\section{Lutzomyia longipalpis (Diptera, Psychodidae) em Cuesta Basáltica, na bacia hidrográfica do Rio Corumbataí, Região Centro-leste do Estado de São Paulo}

\section{Lutzomyia longipalpis (Diptera, Psychodidae) in the Basaltic Cuesta at the hydrographic basin of the Corumbataí River, Central East Region of the State of São Paulo, Brazil}

\section{André Antonio Cutolo' \\ Denis Adriano Camargo'}

Adriano Antonio Cutolo ${ }^{2}$

Claudio José Von Zuben ${ }^{1}$

Eunice Aparecida Bianchi Galati ${ }^{3}$

'Departamento de Zoologia do Instituto de Biociências da UNESP.

${ }^{2}$ Instituto de Geociências da UNICAMP.

${ }^{3}$ Departamento de Epidemiologia da Faculdade de Saúde Pública da USP.
A incidência das leishmanioses tegumentar e visceral americanas, em especial esta última (LVA), em hospedeiros caninos e humanos, encontra-se em crescente processo de expansão no Estado de São Paulo. Para a vigilância epidemiológica dessas endemias, torna-se fundamental o conhecimento da distribuição e da ecologia das diferentes espécies da fauna flebotomínea vetoras. Assim, a divulgação de novos encontros de seus vetores, sobretudo da Lutzomyia longipalpis, o principal vetor da LVA, é fundamental para apontar novas áreas de risco para a transmissão dessas doenças. Neste estudo, capturas de flebotomíneos foram realizadas em ambiente domiciliar, peridomiciliar e de mata, em diferentes localidades rurais dos municípios de Ipeúna e Itirapina, entre outubro de 2001 e fevereiro de 2004. Foram utilizadas armadilhas luminosas automáticas do tipo CDC, das $18 \mathrm{~h}$ às $8 \mathrm{~h}$, em 14 noites, resultando 420 horas de exposição. Foram capturados 177 flebotomíneos pertencentes a doze espécies. A espécie mais abundante, Nyssomyia neivai, apontada como a principal vetora de LTA no Estado, contribuiu com $85,4 \%$ dos espécimes capturados em Ipeúna. O encontro de Lutzomyia longipalpis em uma caverna em Itirapina, aponta para o risco de estabelecimento da LVA na área e a necessidade de mais estudos locais sobre sua ecologia, sobretudo em relação à ocupação de ambientes antrópicos.

Palavras-chave: Leishmaniose. Ecologia de vetores. Epidemiologia. Transmissão. Zoonose. Flebotomíneo. 


\section{Abstract}

Cutaneous (LTA) and Visceral (LVA) American Leishmaniasis incidence is increasing in human and canine hosts, especially LVA, which is expanding throughout the State of São Paulo. Knowledge of the distribution and ecology of different sand fly species is essential for leishmaniasis epidemiological surveillance. The communication of new findings of vectors, mainly the main LVA vector Lutzomyia longipalpis, is mandatory for determination of these illnesses' transmission risk. In this study, sand flies were trapped in domiciliary, peridomiciliary and bushed areas, in different localities in rural areas of the Ipeúna and Itirapina counties, between October 2001 and February 2004. CDC automatic light traps were used from $18 \mathrm{~h}$ to $8 \mathrm{~h}$, during 14 nights, resulting in 420 hours of captures. A total of 177 specimens pertaining to twelve different species were sampled. The most abundant species was Nyssomyia neivai, pointed out as the main LTA vector in the State of São Paulo, which totaled $85.4 \%$ of the specimens trapped in Ipeúna. The finding of Lutzomyia longipalpis in a cave in Itirapina indicates the risk of LVA establishment in the area and the need for new local studies on its ecology, especially with regard to occupation of anthropic environments.

Keywords: Leishmaniasis. Vector ecology. Epidemiology. Transmission. Zoonosis. Sand flies.

\section{Notas e Informações}

ALeishmanioseVisceral Americana (LVA) é uma zoonose em franca expansão no Estado de São Paulo. De 1999 até setembro de 2006, 771 casos humanos da doença foram diagnosticados, com registro de 79 óbitos. A enfermidade já foi diagnosticada em 35 municípios pertencentes a quatro regionais de saúde (DIR) distintas ${ }^{1}$.

Lutzomyia longipalpis é considerada a principal vetora da LVA no Brasil e o conhecimento da sua distribuição no território paulista é fundamental para o controle desta enfermidade. No Estado de São Paulo, sua presença foi inicialmente assinalada nos municípios de Pirapora do Bom Jesus, Cássia dos Coqueiros, Espírito Santo do Pinhal, Itupeva e Socorro, sendo encontrada no ano de 1997 em área urbana consolidada do município de Araçatuba ${ }^{2}$ e posteriormente em 22 municípios do oeste paulista ${ }^{3}$.

No presente estudo, as atividades de levantamento de flebotomíneos tiveram início em uma propriedade situada em área rural no Bairro Quilombo, município de Ipeúna (SP). O local é cortado pelo Ribeirão Passa Cinco, afluente do Rio Corumbataí, e se localiza na Depressão Periférica do Estado, onde localmente o relevo é colinoso, predominando baixas declividades (até 15\%) com amplitudes inferiores a 100 metros $^{4}$.

Para a captura dos insetos, armadilhas automáticas luminosas do tipo CDC foram posicionadas a uma distância de 30 a 50 $\mathrm{cm}$ do solo e funcionaram por um período ininterrupto das 18 às 8 horas da manhã do dia seguinte, tendo sido realizadas 11 coletas, uma em outubro de 2001 e as demais realizadas com freqüência mensal, de fevereiro a novembro de 2003. Em outubro de 2001, foram realizadas coletas no intradomicílio e duas outras às margens do Ribeirão Passa Cinco, em uma estreita área de 5 metros de mata ciliar nativa. Nas demais coletas as armadilhas foram instaladas em um galinheiro situado no peridomicílio, a cinco metros de uma das residências $\left(22^{\circ} 29^{\prime} 24,5^{\prime \prime}\right.$ Sul; $47^{\circ} 38^{\prime} 42,7^{\prime \prime}$ Oeste e 529 metros de altitude), e outra às margens do já citado ribeirão (22²9'32,4"Sul; 47³8'35" 
Oeste e 513 metros de altitude).

Em janeiro e fevereiro de 2004, capturas foram realizadas no intradomicílio, e em galinheiro no peridomicílio, em outra propriedade, vizinha à anterior $\left(22^{\circ} 29^{\prime} 23^{\prime \prime}\right.$ Sul; 47 $38^{\prime} 34,5^{\prime \prime}$ Oeste e 525 metros de altitude).

No mês de maio de 2004, foi feita uma coleta em uma terceira localidade, no município de Itirapina, situada na Fazenda São João da Lapa $\left(22^{\circ} 25^{\prime} 04,7\right.$ ”Sul; $47^{\circ} 47^{\prime} 32,8^{\prime \prime}$ Oeste e 808 metros de altitude). O local se encontra a $17 \mathrm{~km}$ dos outros dois mencionados anteriormente. A propriedade é cortada pelo Córrego da Lapa, divisa entre os municípios de Ipeúna e Itirapina e afluente do Ribeirão Cantagalo, que por sua vez é afluente do Ribeirão Passa Cinco. O local de captura situa-se na Cuesta Basáltica da Serra de Itaqueri, onde o relevo é escarpado predominando declividades altas (maiores que $30 \%$ ) e amplitudes maiores que 100 metros $^{4}$.

Uma armadilha luminosa do tipo CDC foi colocada em área de mata nativa, ao lado de um paredão de arenito de 50 metros de altura e outra, entre as frestas de rochas de uma caverna popularmente conhecida como "Boca do Sapo", com 40 metros de abertura e aproximadamente 100 metros de profundidade.
As coordenadas dos locais de captura foram obtidas por meio de aparelho GPS Garmin Etrex (Global Positioning System) e posteriormente plotadas em mapas topográficos da região. Nas duas primeiras localidades, foram totalizadas 14 noites de coleta, resultando em 420 horas de captura. A identificação dos insetos foi feita segundo Galati ${ }^{5}$.

Nyssomyia neivai mostrou-se a espécie mais abundante nos diferentes locais de captura, correspondendo a $85,38 \%$ do total de espécimes coletados (Tabela 1). A abundância desta espécie no interior paulista é fato conhecido, sendo esta espécie considerada como a principal transmissora da LTA no Estado ${ }^{6}$.

Entre as frestas de rochas do paredão de arenito foram coletadas duas fêmeas de Lutzomyia longipalpis, uma fêmea de Evandromyia sallesi e uma fêmea de Mycropigomyia ferreirana. Entre as rochas da entrada da caverna "Boca do Sapo" situada em meio à mata, no mesmo paredão e à cerca de 50 metros do local anterior, foi coletada uma fêmea de Evandromyia sallesi e um macho de Migonemyia migonei.

Nas capturas realizadas no município de Ipeúna, $N$. neivai, até recentemente considerada na sinonímia de $N$. intermedia, ambas apontadas como as principais

Tabela 1 - Total de flebotomíneos capturados em área rural peridomiciliar do Bairro Quilombo, município de Ipeúna, SP, por espécie, sexo e local da armadilha (outubro de 2001 a fevereiro de 2004).

Table 1 - Total of sand flies captured in a peridomiciliary rural area of the Quilombo borough, Ipeúna county, São Paulo State, Brazil, by species, gender, and trap site (October 2001 to February 2004).

\begin{tabular}{lcccccccccc}
\hline & \multicolumn{2}{c}{ Mata } & \multicolumn{2}{c}{ Galinheiro } & \multicolumn{2}{c}{ Intra-domicílio } & \multicolumn{2}{c}{ Total } & \multicolumn{2}{c}{ Total } \\
Espécie & $\mathrm{F}$ & $\mathrm{M}$ & $\mathrm{F}$ & $\mathrm{M}$ & $\mathrm{F}$ & $\mathrm{M}$ & $\mathrm{F}$ & $\mathrm{M}$ & $\mathrm{F}+\mathrm{M}$ & $\%$ \\
\hline Nyssomyia neivai & 88 & 18 & 21 & 13 & 4 & 2 & 113 & 33 & 146 & 85,38 \\
Nyssomyia whitmani & 6 & - & 1 & - & 2 & - & 9 & - & 9 & 5,26 \\
Pintomyia monticola & 5 & - & - & - & - & - & 5 & - & 5 & 2,92 \\
Brumptomyia brumpti & 2 & 1 & - & - & - & 1 & 2 & 2 & 4 & 2,34 \\
Evandromyia lenti & 2 & - & 2 & - & - & - & 4 & - & 4 & 2,34 \\
Pintomyia pessoai & 1 & - & - & - & - & - & 1 & - & 1 & 0,58 \\
Sciopemyia sordellii & - & - & 1 & - & - & - & 1 & - & 1 & 0,58 \\
Evandromyia cortelezzii & - & - & - & - & - & 1 & - & 1 & 1 & 0,58 \\
Total & 103 & 19 & 25 & 13 & 6 & 4 & 135 & 36 & 171 & 100 \\
\% & 60,23 & 11,11 & 14,62 & 7,6 & 3,51 & 2,34 & 78,94 & 21,05 & 100 & \\
\hline
\end{tabular}

F:fêmeas; M: machos: F+M: soma de fêmeas e machos.

F: females; M: males; F+M: total of females plus males. 
vetoras da LTA no Estado ${ }^{7}$, predominou absolutamente, principalmente na mata ciliar, seguida pela $N$. whitmani, também um outro importante vetor da LTA no Brasil ${ }^{8}$, o que pode ser indicativo deste ambiente como um importante local de risco para a transmissão de LTA.

O encontro de L. longipalpis em área de mata no município de Itirapina, associada às Cuestas Basálticas com afloramentos rochosos arenítico-basálticos, aponta para um habitat silvestre da espécie na região. Embora apenas uma captura tenha sido feita na região da caverna, as espécies encontradas não foram capturadas nas 14 coletas realizadas em área antrópica e de mata ciliar do município vizinho. Face à expansão da
LVA no Estado, faz-se necessária e urgente uma avaliação da possibilidade da mesma se dispersar para ambientes antrópicos, em locais onde esta forma de relevo está presente, pois as Cuestas Basálticas cortam transversalmente o centro do Estado de São Paulo, indo do Rio Grande até o Rio Paranapanema, sendo uma das mais marcantes feições do relevo paulista e caracterizadas pela presença de relevo escarpado nos limites com a Depressão Periférica ${ }^{4}$.

Aprovado pelo Comitê de Ética na Experimentação Animal da Universidade Estadual de Campinas.

Os autores, através de documentação assinada e enviada a RBE, declaram não existir nenhum tipo de conflito de interesses.

\section{Referências}

1. CVE. Casos Autóctones e Óbitos de LVA, segundo Município de Residência, 1999 - 2006. Situação da Leishmaniose no Estado de São Paulo. Disponível em: http://www.cve.saude.sp.gov.br/htm/zoo/lvah_ auto9904.htm [Acessado em 15/11/2006].

2. Costa AIP, Casanova C, Rodas LAC, Galati EAB. 1997. Atualização da distribuição geográfica e primeiro encontro de Lutzomyia longipalpis em área urbana no Estado de São Paulo, Brasil. Rev Saude Publica 1997; 31; 632-33.

3. SUCEN. Distribuição da Lutzomyia longipalpis na região oeste do Estado de São Paulo - Situação até 30/12/00. Boletim LVA - 006. Disponível em: http:// www.sucen.sp.gov.br/doencas/index.htm [Acessado em $15 / 11 / 2006]$.

4. Ponçano WL, Carneiro CDR, Bistrichi CA, Almeida FFM, Prandini FL. Mapa Geomorfológico do Estado de São Paulo. Vol. 1. São Paulo: Divisão de Minas e Geologia Aplicada, Instituto de Pesquisas Tecnológicas do Estado de São Paulo; 1981.
5. Galati EAB. Morfologia, terminologia de adultos e identificação dos táxons da América. In: Rangel EF, Lainson R. Flebotomíneos do Brasil. Rio de Janeiro: Fiocruz; 2003. p. 53-175.

6. Camargo-Neves VLF, Gomes AC, Antunes JLF. Correlação da presença de espécies de flebotomíneos (Diptera: Psychodidae) com registros de casos de leishmaniose tegumentar americana no Estado de São Paulo, Brasil. Rev Soc Bras Med Trop 2002; 35; 299-306.

7. Marcondes CB. Redescription of Lutzomyia (Nyssomyia) intermedia (Lutz \& Neiva, 1912) and resurrection of L. neivai (Pinto, 1926) (Diptera, Psychodidae, Phlebotominae). Mem Inst Oswaldo Cruz 1996; 91; 457-62.

8. Rangel EF, Lainson R. Transmissores da leishmaniose tegumentar americana. In: Rangel F, Lainson $\mathrm{R}$ Flebotomíneos do Brasil. Rio de Janeiro: Fiocruz; 2003. p. 291-305.

Recebido em: 15/01/07 Aprovado em: 27/02/08 